\title{
Wie viel Spezialisierung braucht die Psychotherapie?
}

In der Psychotherapie zeichnet sich eine zunehmende Spezialisierung ab. Spezialisierung wird oftmals als Merkmal einer positiven Weiterentwicklung und Ausdifferenzierung gesehen. Manchmal beziehen sich diese Spezialisierungen auf Neuentwicklungen aus der Psychotherapieforschung, manchmal auch auf die (vermeintlich?) zu wenig gelehrte Vertiefung oder spezialisierte Anwendung. Während früher Gruppen-, Paar- oder Familientherapie ein integraler Bestandteil sowohl in verhaltenstherapeutischen als auch in psychodynamischen Ausbildungen waren, wird sie nun als eine eigenständige Therapieform (mit eigenen Supervisoren, Ausbildungscurricula, privaten Ausbildungsinstituten) geführt. Diese Entwicklungen führen dazu, zum einen eigene, neue Therapieverfahren für die Psychotherapieausbildung, zum anderen aber auch Weiterbildungen, die zusätzlich zur Psychotherapieausbildung absolviert werden sollen, zu fordern. Diverse Curricula wurden ausformuliert, um spezialisierte Expertise zu erlangen (z.B. DBT-Therapeut, EMDR-Therapeut, Psychotraumatologe, Psychodiabetologe, Psychoonkologe, ACTTherapeut). Es stellt sich zunehmend die Frage, ob ein grenzenloses Wachstum von Spezialisierungsanforderungen wirklich sachdienlich ist. Wie viel Spezialisierung ist notwendig? Ab wann wird Spezialisierung zum Hindernis und zur Abwertung einer profunden Grundausbildung? Dieses Thema erscheint uns besonders geeignet für eine Pro-Contra-Diskussion in der Zeitschrift VerHALTENSTHERAPIE. Als Vertreter der beiden Sichtweisen konnten wir akquirieren: Prof. Dr. M. Berking, bekannt als Vertreter transdiagnostischer Therapieansätze, insbesondere von Emotionsregulationstrainings, und Prof. Dr. T. Fydrich, Sprecher der Fachgruppe klinische Psychologie und Psychotherapie, der sich insbesondere für eine spezifische Weiterbildung in "Somatopsychologie» (z.B. zur Behandlung von Syndromen wie chronischer Schmerz, Diabetes mellitus, Tinnitus, gastrointestinale Erkrankungen) einsetzt.

Winfried Rief, Marburg

\section{Pro}

Störungs- und problembezogene Spezialisierung in der Psychotherapie ist ein Zeichen von

Professionalisierung

Fast so alt wie die Psychotherapie selbst und mehr als ein halbes Jahrhundert alt ist auch die Debatte um den Anteil von spezifischen und unspezifischen Wirkfaktoren in der Psychotherapie. Immer wieder wird das mittlerweile schon in die Jahre gekommene Kuchendiagramm von Michael Lambert präsentiert, welches suggeriert, dass der Erfolg psychotherapeutischer Behandlungen nur zu einem geringen Teil auf das spezifische Interventionsverfahren zurückgeführt werden kann. Die «große Psychotherapiedebatte» wurde in der letzten Dekade von Bruce Wampold im gleichen Tenor wieder aufgegriffen bzw. mit einiger Energie und neu aufgearbeiteten statistischen Analysen fortgesetzt. Vergessen wird dabei immer wieder die Varianz, die auf die Interaktion zwischen störungsspezifischen, verfahrensbezogenen und persönlichen Variablen des Therapeuten und Patienten zurückgeführt werden muss.

Hintergrund dieser Debatte ist die auch immer wieder aufflackernde Diskussion um Vor- und Nachteile des «medizini-

\section{KARGER}

Fax +49 7614520714

Information@Karger.de

www.karger.com (c) 2011 S. Karger GmbH, Freiburg

1016-6262/11/0211-0061\$38.00/0

Accessible online at:

www.karger.com/ver 
schen Modells» bzw. des «psychosozialen Modells» psychischer Störungen und ihrer Behandlung. Die «Ganzheitlichkeit» und die «Methodenvielfalt» gelten als oft bemühte vermeintliche Qualitätsstandards für psychotherapeutische Interventionen im Einzelfall oder gar für das psychotherapeutische Versorgungssystem insgesamt.

Dem steht die umfangreiche Weiterentwicklung der Grundlagenforschung zu psychischen Störungen und zu körperlichen Erkrankungen gegenüber, bei denen psychische Faktoren eine bedeutsame Rolle bei Entstehung, Aufrechterhaltung und Rehabilitation spielen. Zusätzlich steht dieser Position auch eine fortlaufende Weiterentwicklung der Störungsmodelle und der Behandlungsansätze entgegen. Diese erfolgt unter Berücksichtigung der Erkenntnisse epidemiologischer, psychologischer, biologischer und sozialer Befunde.

Dem «Universalitätsmythos» («alle psychotherapeutischen Ansätze helfen bei allen Störungen») gegenüber stehen auch die eindeutige Entwicklung und der hohe Grad an Sorgfalt, mit dem seit einigen Jahren Leitlinien (auch) für die Behandlung psychischer Störungen entwickelt werden. Selbstredend sind nahezu alle Leitlinien störungsbezogene Leitlinien. Dokumente, die eindeutig für störungsspezifische und störungsfokussierte (psychotherapeutische) Behandlungen sprechen, sind zahlreiche Ansätze und Publikationen, deren Titel jeweils schon Programm sind:

Im angloamerikanischen Sprachraum sind dies beispielsweise «What Works for Whom» [Roth and Fonagy, 2005], «A Practitioners's Guide to Evidence-Based Psychotherapy» [Fisher, 2006], «A Guide to Treatments That Work» [Nathan und Gorman, 2002], der Ansatz der «evidence-based practices in mental health» oder der «empirically supported therapies» (EST).

Auch im deutschen Sprachraum lässt sich der störungsbezogene Ansatz in der aktuellen Fachliteratur klar erkennen. In der so genannten blauen Reihe, Fortschritte der Psychotherapie, (Hogrefe Verlag) sind bereits 43 störungsspezifische, praxisorientierte Büchlein mit Manual-Charakter erschienen, in denen jeweils die praxisrelevanten Grundlagenkenntnisse und (verhaltenstherapeutischen) Interventionsbausteine für die jeweilige Störung dargestellt und erläutert werden.

Es ist wenig überraschend, dass vor allem im verhaltenstherapeutischen «Lager» der störungsspezifische Ansatz weit verbreitet ist. Es ist jedoch bisher weniger bekannt, dass seit einigen Jahren auch psychodynamisch arbeitende Kolleginnen und Kollegen sowie «die Systemiker» und auch die Gesprächspsychotherapeuten (oder solche, die der Gesprächstherapie nahestehen) mehr und mehr den Ansatz der weitgehend auf interpersonelle Beziehung oder das Verstehen aufgebauten Therapietheorie um eine klar störungsspezifische, teilweise sogar manualisierte Komponente bzw. Sichtweise erweitern. So erschien kürzlich das erste Bändchen einer Reihe zur störungsorientierten und manualisierten psychodynamischen Psychotherapie [Beutel et al., 2010]. Der zweite Band des Lehrbuchs «Systemische Therapie und Beratung» von J. Schweitzer und A. von Schlippe [2007] trägt den Untertitel: «Das störungsspezifische Wissen», und Finke und Teusch entwickelten für verschiedene psychische Störungen Manual-geleitete Therapieanleitungen auf der Grundlage der Gesprächspsychotherapie [z.B. Finke und Teusch, 1999].

\section{Eine Depression ist keine Panikstörung ist keine Essstörung ...}

Die spezifischen bekannten und erforschten Grundlagen und Modelle psychischer Störungen (oder somatischer Erkrankungen) bei der Behandlung von Personen mit ebensolchen Störungen zu ignorieren wäre und ist grob unprofessionelles Verhalten. «State-of-the-art»-Behandlungen erfordern ein fortlaufend aktualisiertes Wissen über die biologischen (medizinischen), psychologischen und sozialen Erkenntnisse bzw. Befunde zu eben diesem Störungsbild. Auf die unspezifischen Aspekte der Behandlung allein zu vertrauen würde bedeuten, die wissenschaftlichen Erkenntnisse zu den jeweiligen Störungen nicht zu beachten. Die verhaltensrelevanten circadianen Rhythmen im endokrinen System bei Depressiven unterscheiden sich beispielsweise massiv von psychophysiologischen Abläufen während eines Panikanfalls oder den somatischen und psychischen Folgen massiver Unter- oder Fehlernährung, wie sie bei Personen mit Essstörungen häufig vorkommen. Das Verhalten eines chronischen Schmerzpatienten mit Analgetika-Abusus ist unbedingt zu unterscheiden vom Drogengebrauch eines Junkies oder vom Trinkverhalten eines «Spiegeltrinkers». Sowohl der Serotoninhaushalt als auch die spezifischen Ängste und das Verhalten eines Zwangserkrankten mit massiven Kontaminationsängsten sind gänzlich anders zu erklären als eine möglicherweise stark hormonell bedingte rezidivierende Depression bei einer betroffenen Person, bei der zudem psychotische Wahninhalte auftreten. Niemand würde einen Internisten wählen, der die Gefahreneinschätzung für die Entwicklung eines Diabetes auf der Grundlage des Blutbildes nicht auf der Grundlage des aktuellen Stands der Wissenschaft einzuschätzen weiß oder der nicht in der Lage ist, eine spezifische weiterführende Diagnostik (und dann auch eine entsprechende Behandlung) zu initiieren, wenn die Befunde noch nicht ausreichen, um ein akutes Beschwerdebild zu erklären und zu behandeln. Ebenso würde niemand mit zunehmend schlimmer werdenden Knieproblemen freiwillig zu einem Orthopäden gehen, der nicht über die Möglichkeiten moderner Operations- oder anderer Interventionsmethoden informiert ist. Bei lang andauernden unerklärlichen Schulterschmerzen ist das Aufsuchen eines Schulterspezialisten zwar keine Garantie für eine erfolgreiche Behandlung; die Wahrscheinlichkeit, die bestmögliche Behandlung zu erhalten, ist jedoch dann sicher am größten.

Will sagen: Auch für den Bereich der Psychotherapie ist es «evident», dass von einem Therapeuten, der für den jeweili- 
gen Bereich gut ausgebildet ist und der neben einer fundierten (akademischen) Ausbildung auch umfassende klinische Erfahrungen mitbringt, die bestmögliche Behandlung erwartet werden kann. Psychotherapeuten sind keine «Generalisten». Sie haben hinsichtlich der Behandlung von Personen mit unterschiedlichen Beschwerden und Erkrankungen unterschiedlich gute Ausbildungen und unterschiedlich umfassende Erfahrungen. Das Verstehen allein oder eine ausschließlich interpersonelle Perspektive bei der psychotherapeutischen Behandlung von Personen ist jedoch keineswegs ausreichend für eine professionelle Psychotherapie. Der aktuelle Stand der Wissenschaft hinsichtlich Störungskenntnis, Diagnostik und Behandlung muss unbedingt berücksichtigt werden.

Zudem muss und kann das Ziel psychotherapeutischer Behandlungen im Gesundheitssystem nur sein, die bestmögliche Behandlung anzubieten. Antidepressiva werden verordnet, obwohl die unspezifischen Wirkfaktoren doppelt so viel Varianz erklären wie die spezifischen [Rief et al., 2009]. So muss auch für die Psychotherapie gelten, das Optimale anzubieten: egal, ob die spezifischen Faktoren und Kompetenzen 10 oder $60 \%$ der Erfolgsvarianz von Behandlungen erklären! Dafür braucht man aber spezifisches Störungswissen und eine auf die Diagnose zugeschnittene Behandlungskompetenz.

\section{Ist jedoch eine formalisierte Spezialisierung (in Form von «Titelsammeln») notwendig?}

Vorerst bleibt die Frage, ob es für alle Störungen (oder für Störungsgruppen) eine formalisierte Spezialisierung im Sinne einer mehr oder weniger umfangreichen Aus-, Fort- oder Weiterbildung braucht oder nicht. Hierzu ist zu sagen, dass die aktuellen Ausbildungsgänge - Diplom (bzw. Master) in Psychologie, mindestens 3-jährige Psychotherapieausbildung mit Praxiszeiten in Psychiatrie, Psychosomatik und in der Ausbildungsambulanz inklusive Supervision, Selbsterfahrung und der theoretischen Ausbildung - für eine umfassende Qualifikation zur psychotherapeutischen Behandlung aller Störungen, bei denen Psychotherapie indiziert ist, klar ausreichen. Personen, die diese Ausbildung erfolgreich abgeschlossen haben, haben nach minimal 8 Jahren ein Ausbildungsniveau, das dem eines Facharztes vergleichbar ist. Für einzelne Bereiche, deren Vertiefung in der psychotherapeutischen Ausbildung nicht ausreichend möglich ist, sind jedoch (formalisierte) Weiterbildungen oder zumindest (curriculare) Fortbildungen angemessen. Dies gilt beispielsweise für die klinische Neuropsychologie oder den Schwerpunkt der klinischen Somatopsychologie (derzeit als Entwurf in der Musterweiterbildungsordnung der Bundespsychotherapeutenkammer in Diskussion) als einen Bereich der psychotherapeutischen (Ko-)Behandlung bei schweren oder chronischen somatischen Erkrankungen. Auch ist für die qualifizierte Behandlung von Personen verschiedener Altersgruppen (Kinder, Jugendliche, Erwachsene, ältere Erwachsene) in einem Mindestumfang der
Erwerb spezifischer Kenntnisse und Fertigkeiten im Rahmen von Fort- oder Weiterbildungen angemessen.

Nicht angemessen und lediglich als «Kür» (manchmal aber auch als «Kontamination» der Herkunftszunft) zu betrachten sind jedoch die allseits sprießenden Angebote verschiedenster vermeintlich besonderer Therapieansätze, deren Evidenzbasierung keineswegs den teilweise zu beobachteten «Hype» rechtfertigt - sei es die Traumtherapie (jedweder Couleur inklusive EMDR) oder seien es Schematherapie, Hypnotherapie, akzeptanzbasierte Therapieansätze oder die dialektisch-behaviorale Therapie für Borderline-Störungen. Die genannten Ansätze enthalten dabei durchaus klinisch gut nachvollziehbare Ansätze und Interventionstechniken, die sich meist auch mit den klassischen Therapieansätzen «vertragen» (zumindest mit der Verhaltenstherapie). Von der gesamten Profession zu fordern bzw. zumindest zu wünschen wäre es, dass die als gut wirksam nachgewiesenen und somit evidenzbasierten Interventionsmethoden oder -techniken auch entsprechend enthusiastisch in der Praxis umgesetzt und eingesetzt werden. Ein individueller «Eklektizismus» in der Therapeutenschaft, der sich häufig nach jedem Fortbildungswochenende neu einpendelt und oft als «ganzheitlich» oder «methodenvielfältig» gepriesen wird, ist jedenfalls nicht der richtige Weg in eine gewinnbringende «Spezialisierung».

Thomas Fydrich, Berlin

\section{Literatur}

Beutel ME, Doering S, Leichsenring, F, Reich G: Psychodynamische Psychotherapie - Störungsorientierung und Manualisierung in der therapeutischen Praxis. Göttingen, Hogrefe, 2010.

Finke J, Teusch L: Entwurf zu einer manualgeleiteten Gesprächspsychotherapie der Depression. Psychotherapeut 1999;44:101-107.

Fisher JE: Practitioner's Guide to Evidence-Based Psychotherapy. Berlin, Springer, 2006.

Nathan PE, Gorman JM: A Guide to Treatments That Work, ed 2. New York, Oxford University Press, 2002

Rief W, Nestoriuc Y, Weiss S, Welzel E, Barsky AJ, Hofmann SG: Meta-analysis of the placebo response in antidepressant trials. J Affect Dis 2009;118:1-8.

Roth A, Fonagy P: What Works for Whom? A Critical Review of Psychotherapy Research, ed 2. New York, Guildford Press, 2005.

Schweitzer J, von Schlippe A: Lehrbuch der systemischen Therapie II - das störungsspezifische Wissen, ed 2. Göttingen, Vandenhoeck \& Ruprecht, 2007.

\section{Contra}

\section{Eine Ode an die Verhältnismäßigkeit und ein Plädoyer für therapeutische Metakompetenzen}

«Hört das denn nie auf? Muss ich nach a) 5-jährigem Studium der Psychologie, in dem ich mich intensiv mit dem Erklären und Verändern menschlichen Erlebens und Verhaltens beschäftigt und in dem ich im Bereich Klinische Psychologie 5 
Seminare, 3 Vorlesungen, 3 Prüfungen, eine Fallarbeit und diverse Praktika absolviert habe, b) 600 Theoriestunden postgradualer Weiterbildung zum Psychologischen Psychotherapeuten (PPT), c) 600 im Rahmen der Ausbildung geleisteten Behandlungsstunden, d) 600 Psychosomatikstunden, e) 1200 Behandlungsstunden im Rahmen des Psychiatriejahres, f) 120 Stunden Selbsterfahrung, g) 150 Stunden Supervision im Rahmen der Ausbildung, h) weiteren Supervisions- und Intervisionsstunden an meinen diversen Arbeitsplätzen, i) zahlreichen zusätzlichen Workshops, die ich aus Interesse besucht habe, und j) nicht zuletzt den vielen Therapien, die ich mittlerweile mit viel Einsatz und Engagement absolviert habe, jetzt noch einmal viel Geld und Zeit in die Zusatzausbildung zur DBT-/Familien-/Paar-/EMDR-/MBCT-/ACT-etc.-Therapeutin investieren, weil ich nur dann ausreichend qualifiziert für meinen Job bin? Was ist eigentlich in meiner vorausgehenden Aus- und Fortbildung passiert, wenn sie mich trotz ihres Umfangs anscheinend nicht ausreichend qualifiziert hat? Werde ich durch eine weitere Zusatzqualifikation wirklich zu einer besseren Therapeutin? Oder diene ich nur als weitere Einkommensquelle der Fortbildungsinstitute?»

So oder so ähnlich mögen sie lauten, die Fragen, die engagierten klinischen Psychologinnen und Psychologen auf Stellensuche durch den Kopf gehen, wenn sie Ausschreibungen lesen, die mit dem Zusatz enden: «Eine abgeschlossene oder begonnene Weiterbildung zum/zur DBT-etc.-Therapeuten ist erwünscht.» Und zu Recht! Auch wenn ein Mehr an Wissen und Handlungskompetenz prinzipiell immer wünschenswert und für spezifische Anwendungsgebiete erforderlich sein mag, so muss doch der etwaige zusätzliche Gewinn im Verhältnis zu den notwendigen Aufwendungen und Ressourcen stehen, die mit dem Erwerb von Zusatzqualifikationen entstehen.

Die akademische und postgraduale Ausbildung zum PPT ist in Deutschland gemessen am internationalen Standard ausgesprochen umfangreich und gut. Sie misst sich an dem Anspruch, die theoretischen Kompetenzen zu vermitteln, die für den effektiven Einsatz der erfolgversprechendsten Verfahren bei sämtlichen psychischen Störungen notwendig sind. Aufgrund ihres Umfangs lässt sich postulieren, dass bei entsprechender Gestaltung ausreichend Möglichkeiten geboten werden können, diesen Anspruch zu erfüllen. Sämtliche Interventionsverfahren, die sich für die Versorgung als relevant erweisen, sollten in allererster Linie im Rahmen der Ausbildung zum PPT vermittelt werden. Vor dem Hintergrund der Relevanz von gruppentherapeutischen Angeboten und Angeboten für Kinder und Jugendliche sollten auch diese Bereiche meines Erachtens Teil der «Grundausbildung zum PPT» sein. Eine zunehmende Fokussierung auf Post-PPT-Spezialisierungen ist dagegen mit der Gefahr verbunden, diese Inhalte aus der PPT-Ausbildung auszulagern, womit die Gewährleistung des störungsübergreifenden Versorgungsanspruchs gefährdet wäre.

Psychotherapeutisches Handeln ist immer eine Mischung von Handwerk und Kunst. Jeder Behandlungsfall ist einzigartig und erfordert die Anpassung empirisch-validierter Behandlungsstrategien an die Bedürfnisse und Fähigkeiten des einzelnen Patienten. Eine zunehmende Spezialisierung von Fortbildungsangeboten, die primär darauf fokussieren, für jede potentielle Problemkategorie konkrete Vorgangsweisen zu vermitteln, muss in Anbetracht der Menge potentiell relevanter Problemkonstellationen als ineffizient eingestuft werden. Stattdessen gilt es, eine angemessene Balance in der Vermittlung empirisch-validierter Verfahren und kreativer Adaptationskompetenzen zu finden. Die Ausbildung zum Psychotherapeuten sollte neben Richtlinien zum Vorgehen bei prototypischen Problemen auch therapeutische Metakompetenzen vermitteln, die dabei helfen, Problembereiche jedweder Art konstruktiv zu analysieren und, aufbauend auf dieser Analyse, vielversprechende Änderungspunkte abzuleiten, individualisierte Behandlungspläne zu erstellen und diese kompetent umzusetzen. Die Schulung im eigenständigen, flexiblen und kreativen Abstimmen von störungsbezogenen und motivationsfördernden Vorgehensweisen, die sich bei ähnlich gelagerten Problemen als effektiv erwiesen haben, an die Erfordernisse des jeweiligen Patienten sollte ein wesentliches Element der Ausbildung zum PPT sein. Nur so ist zu gewährleisten, dass angehende PPTs ihrem Versorgungsauftrag nachkommen können. In dem Maße, in dem Ausbildungskandidaten in der «Grundausbildung zum PPT» neben einem fundierten Störungs- und Veränderungswissens auch effektive Metakompetenzen erwerben, reduziert sich aber auch die Notwendigkeit weiterer und zunehmend spezialisierter Fortbildungen.

Obligatorische Fortbildungsangebote, die über die «Grundausbildung» hinausgehen, haben ihre Berechtigung ausschließlich in relevanten Anwendungsfeldern, in denen nur ein geringer Prozentsatz der ausgebildeten PPTs arbeitet und in denen die Erfordernisse jenseits dessen liegen, was in der Ausbildung üblicherweise vermittelt wird. In diesem Sinne lässt sich argumentieren, dass beispielsweise Patienten mit Borderline-Persönlichkeitsstörung (BPS) eine besonders relevante Patientengruppe darstellen, die sich durch ein ausgesprochen intensives Leiden und durch das Verursachen von hohen Kosten im Gesundheitssystem auszeichnen und deren Behandlung für jeden Psychotherapeuten eine große Herausforderung darstellt. Ungenügend qualifizierte Therapeuten können bei diesen Patienten unter Umständen mehr schaden als nutzen, was vor dem Hintergrund der hohen Suizidrate in dieser Störungsgruppe besonders berücksichtigt werden sollte. Es lässt sich weiter argumentieren, dass im Studium primär theoretisches Wissen über BPS vermittelt wird und dass konkrete therapeutische Vorgehensweisen in der Ausbildung zum PPT in der Regel überwiegend oder ausschließlich in der Arbeit an anderen Störungsbildern erfolgt. Wenn sich unter den obligatorischen 6 Ausbildungsfällen kein BPS-Patient befindet, erscheint es unwahrscheinlich, dass die Expertise erworben wird, die für den täglichen Umgang mit hochgradig belasteten BPS-Patienten notwendig ist. Vor diesem Hintergrund erscheint eine zusätzliche Fortbildung in speziell 
auf BPS zugeschnittenen und erfolgreich evaluierten Therapieverfahren wie DBT für Therapeuten, die primär oder ausschließlich mit diesem Klientel arbeiten, sinnvoll. Ähnliches mag auch für die Arbeit mit Autisten oder schwer traumatisierten Patienten gelten. Die Berechtigung spezifischer Weiterbildungsinhalte sollte jedoch transparent von unabhängigen Experten auf der Grundlage von Daten zur Versorgungslage, zur Effektivität verfügbarer Verfahren und zum Aufwand der Schulung in diesen Verfahren eingeschätzt werden.

In der aktuellen Praxis ist ein in diesem Sinne ausgewiesener Spezialbedarf allerdings nur einer der Gründe für die Existenz von Post-PPT-Weiterbildungen. Zu den ebenfalls in der Weiterbildungspraxis relevanten, aber - meines Erachtens nicht legitimen - Gründen zählt aber auch, dass einige Entwickler innovativer Verfahren ein exklusives Recht auf die Schulung und Zertifizierung in diesen Verfahren beanspruchen. Auch wenn dieses in der Regel mit Qualitätssicherungsaspekten begründet wird, lässt sich vermuten, dass bei solchen Strategien auch finanzielle Interessen eine wesentliche Rolle spielen. In der Folge können dann diese neuen Interventionsformen nicht in dem Maße, in dem es wünschenswert wäre, in die etablierten Ausbildungsgänge integriert werden. Solchen Entwicklungen muss Einhalt geboten werden. Die Dissemination effektiver Therapieverfahren darf durch restriktive Handhabe eines «Copyrights» auf diese Verfahren nicht behindert werden.

Neben dem realen Bedarf nach weiterführender Spezialisierung und dem wirtschaftlichen Interesse verschiedener Therapieentwickler gibt es noch einen weiteren wichtigen Grund für die Zunahme von spezialisierten Fortbildungsangeboten: Nach Jahren der Beschäftigung mit vorgegebenen Fortbildungsinhalten hegen viele Therapeuten den Wunsch, mit frei gewählten Fortbildungsangeboten ihre Kompetenzen zu erweitern und damit die tägliche Therapieroutine weiter spannend und interessant zu halten. Da hierbei das Bedürfnis nach Abwechslung und Selbstbestimmung oft eine wesentliche Rolle spielt, sind Fortbildungsinhalte, die nicht Teil der üblichen Ausbildungscurricula oder der sich anschließenden obligatorischen Fortbildungsveranstaltungen sind, besonders attraktiv.

Diese Anliegen und Interessen sind legitim und sorgen dafür, dass es immer einen Markt geben wird für Angebote, die nicht in die Standardausbildung integriert sind. Da Konkurrenz bekanntermaßen das Geschäft belebt, ist gegen einen Markt jenseits der Standardverfahren im Prinzip nichts einzuwenden. Außerdem zeigt sich immer wieder, dass therapeutische Ansätze, die über lange Zeit vom wissenschaftlichen Diskurs ignoriert wurden, bei entsprechender Konzeptualisierung und Forschungsaktivität ihre Effektivität nachweisen konnten und in der Folge auch Anspruch auf Vermittlung im Rahmen der Standardcurricula erheben können (Stichwort: achtsamkeitsbasierte Interventionstechniken). Problematisch wird der Wunsch nach frei wählbaren, exotisch-innovativen Fortbildungsangeboten nur dann, wenn dadurch die Dissemination nicht effektiver Verfahren gefördert wird. Um diese Gefahr zu reduzieren, empfiehlt es sich in der Ausbildung zum PPT und in den sich anschließenden obligatorischen Fortbildungsangeboten eine breite Palette von Verfahren zu thematisieren, ausreichend viele Wahloptionen zu integrieren und vielversprechende innovative Interventionsverfahren möglichst frühzeitig mit in den Themenkatalog aufzunehmen.

Als Fazit lässt sich somit feststellen: Natürlich ist nichts dagegen einzuwenden, wenn gut ausgebildete Psychotherapeuten sich aus freiem Antrieb und Interesse in einer Vielzahl weiterer Verfahren schulen und so über ein «lifelong learning» dafür sorgen, dass sie ihren Beruf nachhaltig mit Neugier, Engagement und dem Gefühl des persönlichen Wachstums verfolgen. Aber: Solche Fortbildungen sollten nicht zum normativen Standard erhoben werden, sondern sollten seltenen und relevanten Anwendungsfeldern vorbehalten bleiben. Welche spezifischen Fortbildungen für welche Anwendungsfelder indiziert sind, sollte transparent und empirisch begründet, und nicht durch finanzielle Interessen von TreatmentEntwicklern bestimmt werden. Die Ausbildung zum PPT ist meines Erachtens lang genug und bietet ausreichend Zeit für die Vermittlung der Kompetenzen, die für eine flächendeckende und störungsübergreifende Versorgung notwendig sind. Durch die Schulung in der kreativen und auf den Einzelfall abgestimmten Nutzung bewährter Therapieprinzipien können angehende Therapeuten effektiv und effizient auf die Vielfalt der sie erwartenden Problemkonstellationen vorbereitet werden.

Matthias Berking, Marburg 